\title{
La 'pasión' como cemento cultural en el periodismo deportivo escrito en la ciudad de México
}

institucionales.us.es/ambitos/

\section{Juan Carlos Colin Vaughan \\ Universidad Iberoamericana Ciudad de México \\ carlos.colin@ibero.mx}

English Version: 'Passion' as cultural cement in sports journalism in Mexico city

Este artículo busca identificar y explicar el funcionamiento de los valores dominantes en la ideología profesional de periodistas deportivos de medios escritos localizados en la Ciudad de México. Para ellos, el valor central es denominado "pasión", lo que habla de un vínculo emocional entre el periodista deportivo y su profesión. Esta noción tiene una función cohesiva que opera como "cemento cultural" en palabras de Deuze (2005) incluso en un contexto de cambios ocasionados en parte por la la

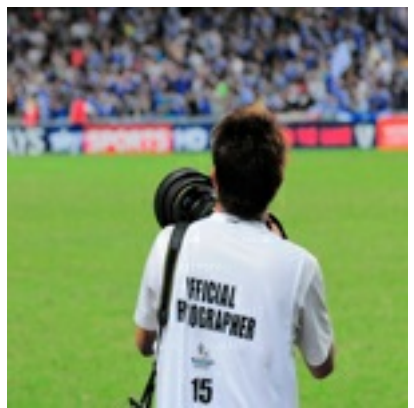
convergencia digital. Este valor dominante en la cultura del periodismo deportivo es un elemento que tiene un papel en la construcción de la identidad profesional para este grupo de periodistas. La metodología del estudio, de enfoque cualitativo, se basó en la realización de 29 entrevistas temáticas semiestructuradas aplicadas a periodistas deportivos de la Ciudad de México.

Palabras clave: periodismo deportivo, ideología profesional, cultura periodística, identidad profesional, valores profesionales, profesionalismo.

Abstract: This article seeks to identify and explain the functioning of the dominant values in the professional ideology of sports journalists of written media located in Mexico City. For them, the central value is called "passion", which shows an emotional link between sports journalists and their profession. This concept has a cohesive function that operates as "cultural cement" in words of Deuze (2005) even in a context of changes caused in part by digital convergence. This dominant value in the culture of sports journalism is an element that has a role in the construction of professional identity for this group of journalists. The methodology of the study, with a qualitative approach, was based on 29 semi-structured thematic interviews applied to sports journalists in Mexico City.

Keywords: sports journalism, professional ideology, journalistic culture, professional identity, professional values, professionalism.

\section{INTRODUCCIÓN}

Por diversas razones, los periodistas deportivos en varias regiones han estado sujetos a estereotipos y prejuicios que consideran su actividad una tarea sencilla e incluso divertida, al grado de que su área ha sido etiquetada como toy department (departamento de juguetes) como refieren algunos 
autores anglosajones (Boyle, 2006; Andrews, 2006; Rowe, 2007; Hardin, Zhong \& Whiteside, 2009; Nicholson, Zion \& Lowden 2011). A menudo, los periodistas deportivos son acusados de comportarse como fans y su trabajo profesional es menospreciado por su distanciamiento hacia temas políticos o sociales y su orientación al mercado, pese a no ser exclusiva de este tipo de periodismo. El escaso prestigio de los periodistas deportivos no es nuevo; en estudios pioneros (Garrison \& Salwen, 1989: 77) se daba cuenta de las críticas que recibía el periodismo deportivo por su poca afinidad con rasgos profesionales clásicos como la "capacitación formal, educación, conocimiento y prácticas únicas, libertad y autonomía". Además, se trata de un periodismo cuyas principales características no suelen estar asociadas a roles como el watchdog (perro guardián), considerado esencial para ejercer un periodismo objetivo por parte de la prensa liberal (English, 2016: 532).

El trabajo que aquí se presenta busca colaborar en cubrir una parte de los vacíos existentes en el conocimiento académico acerca del periodismo deportivo. El objetivo principal del estudio es profundizar en uno de los aspectos que conforman la cultura periodística: la ideología profesional. La noción de cultura periodística es un concepto más amplio que anteriormente también ha sido descrito como newspaper culture (Knott, Carroll \& Meyer, 2002) o culture of news production (Schudson, 2003). Para Hanitzsch (en Oller y Meier, 2012: 31), la cultura periodística engloba el conjunto de principales orientaciones (valores, actitudes y convicciones), prácticas y artefactos (productos y textos) presentes en el trabajo de los profesionales de la información. En otras palabras, la cultura periodística es un concepto que abarca ideas, prácticas y productos noticiosos. De esta forma, se concibe en el estudio que los periodistas deportivos han conformado una cultura periodística propia porque a lo largo de más de un siglo han construido una forma de hacer periodismo que tiene ciertas características que los distinguen de otros periodistas. Una de estas características es el vínculo emocional que tienen con la profesión. Como señala Betts (en Salwen y Garrison, 1998: 88) desde finales del siglo XIX el periodismo deportivo empezó a erigirse como un área particular por distintas razones, como el hecho de tener su propio departamento, estilo de escribir, valores y tradiciones.

A pesar de que en México el periodismo deportivo se gestó en la segunda mitad del siglo XIX (Santiago, 2001) y de que representa un activo importante para los medios de comunicación desde el punto de vista comercial, no existen actualmente suficientes aportes teóricos o empíricos de este tema en el país. En la Ciudad de México se cuenta con una larga historia de medios de comunicación especializados en deportes, empezando por la aparición de la revista The Mexican Sportsman en 1896. Algunas décadas después se fundaron en el país tres de los diarios deportivos más antiguos de América Latina: La Afición (1930), Esto (1941) y Ovaciones (1946). Estos tres fueron los primeros diarios deportivos del país y hasta la fecha siguen circulando en el mercado. Además, hoy estos longevos diarios coexisten con otros periódicos especializados como Récord y Estadio. A estos hay que agregar suplementos y secciones deportivas de diarios populares y de referencia que imprimen decenas de miles de ejemplares al día. La oferta de información deportiva también ha crecido considerablemente en otros soportes como internet, radio y televisión abierta o restringida. La Ciudad de México cuenta además con una estación de radio exclusivamente de deportes, llamada $W$ Deportes, administrada por Televisa y Grupo 
PRISA. Por otra parte, cadenas deportivas internacionales como ESPN y Fox Sports tienen estudios y redacciones en la Ciudad de México desde hace algunos años, para lo cual emplean a periodistas locales y extranjeros.

En esta gran diversidad de medios especializados conviven distintos perfiles de periodistas deportivos, por lo que no se trata de un grupo homogéneo como sugieren los estereotipos que se han construido a su alrededor. Lamentablemente en México no se ha realizado algún estudio sociodemográfico a periodistas deportivos como sí ha ocurrido en países como Australia (Henningham, 1995; Lange, 2002; Nicholson, Zion \& Lowden 2011) o Estados Unidos (Garrison \& Salwen, 1989). Aunque no se conocen las características demográficas con exactitud de los periodistas deportivos en México, a través de estudios cualitativos se reconoce que entre ellos mismos existen distintos perfiles, prioridades, roles profesionales, intereses, backgrounds, estilos, e incluso diversas tensiones y rivalidades. Pero, por otro lado, también es posible observar elementos distintivos que pueden reconocerse en los periodistas deportivos; estos elementos (tangibles o intangibles) tienen un rol en la construcción de una identidad profesional.

\subsection{La metáfora de "cemento cultural” en relación a la identidad e ideología profesional}

Si bien los periodistas forman parte de una cultura heterogénea en la que se manifiestan distintas prácticas y maneras de pensar, al mismo tiempo existen valores que los unen y dan forma a su identidad. Siguiendo el trabajo sobre identidad profesional de Tajfel, el estudio de Grubenmann y Meckel (2015) parte de la existencia de dos facetas de la identidad: la individual y la social. La segunda de ellas, más cercana a la identidad profesional, es definida como "El reconocimiento del individuo de que él [o ella] pertenece a ciertos grupos sociales junto con algún significado emocional y de valor para él [o ella] de esta membresía grupal" (Tajfel, en Grubenmann y Meckel, 2015: 733). Estos mismos autores argumentan que la identidad profesional se conforma por valores, prácticas y concepciones de rol, por lo que se trata de un concepto más amplio de lo que abarca el presente trabajo, el cual está enfocado únicamente en los valores profesionales, es decir, en uno de los elementos que integran la noción de identidad profesional.

Deuze (2005), quien concibe al periodismo como una ideología más que como una profesión, utiliza la metáfora de "cemento cultural" al hablar de los aspectos intangibles que tienen una función cohesiva. Esta analogía forma parte de la ideología profesional, un concepto que en los estudios sobre periodismo puede ser entendido como "el conjunto de relaciones de los valores, orientaciones y predisposiciones que se articulan asimismo como la cultura profesional dominante" (Hanitzsch, 2007, en Oller \& Meier, 2012: 30). Deuze (2005: 445) agrega que la ideología es un "sistema de creencias característico de un grupo particular". La ideología profesional es uno de los aspectos intangibles que forman parte del nivel evaluativo (Hanitzsch, 2007) de la cultura periodística. Sin embargo, la ideología de tipo profesional no es la única presente en una cultura periodística, ya que en una organización informativa pueden encontrarse otras ideologías de diversos tipos, como la ideología política, social, religiosa, etc. Por otro lado, la ideología generalmente es vista como una fuerza cohesiva, integradora y socializadora (Shoemaker \& Reese, 1991, en Oller \& Meier, 2012: 30). En este sentido, los valores destacados por los periodistas deportivos entrevistados cumplen una función cohesiva entre los integrantes de la 
profesión, al ser parte de la cultura profesional dominante.

Debido a su carácter dinámico, es pertinente decir que la ideología profesional depende del contexto temporal y geográfico. Si bien los periodistas deportivos de la Ciudad de México pueden tener características compartidas, también es posible que en otros países o incluso en ciudades de provincia haya sutiles diferencias en cuanto a la jerarquía de valores, actitudes, roles o predisposiciones. Lo mismo sucede con el contexto temporal; quizás dentro de algunos años se sumen otros valores o bien se modifique el significado de lo que hoy en día los periodistas deportivos conciben como valores centrales. Como advierte Reese (2001), se debe tener cautela al trabajar significados de conceptos básicos a través de distintas culturas, poniendo como ejemplo lo que sucede con el problemático concepto de profesionalismo en el periodismo. De esta forma, los significados de ciertos conceptos deben entenderse en un contexto cultural específico (Reese, 2001: 7).

Por otra parte, autores como Deuze (2005) han reconocido categorías normativas que caracterizan al periodismo. Según este autor en el periodismo hay cinco rasgos clave de la actividad, los cuales dan legitimidad y credibilidad al trabajo de los periodistas. Estos son: servicio público, objetividad, autonomía, inmediatez y ética. La pregunta es si estos elementos descritos por Deuze coinciden con el parecer de los periodistas deportivos de la Ciudad de México. Como advertía Reese, esta clase de significados pueden variar en distintos contextos culturales y es justo lo que sucede con el caso del presente estudio. En el caso de los periodistas entrevistados, no dan demasiada importancia al servicio público, la autonomía o la ética, además de cuestionar la objetividad. En cambio, aspectos subjetivos como el gusto por el deporte, la disciplina y habilidades prácticas parecen ser algunos de los valores más importantes en la ideología profesional del periodismo deportivo en México.

\subsection{Ideología profesional e identidad en un entorno adverso}

Uno de los problemas del periodismo en general es que se ve afectado por una autonomía débil (Bourdieu, en Benson, 2005: 41) en gran medida a causa de las fuertes presiones económicas que se manifiestan en su práctica. Aunado a dicho problema, el estatus de los periodistas deportivos ha sido especialmente cuestionado (Boyle, 2006; English, 2015; Garrison \& Salwen, 1998; Hardin, Zhong y Whiteside, 2009) de manera que la profesión de periodista deportivo posee escasa legitimidad, aunque en algunos países puede variar el nivel de reconocimiento de este periodismo temático (Rowe, 2007). La tendencia hacia la comercialización del periodismo deportivo también acompaña al propio deporteespectáculo, cuyos eventos y competencias mediatizadas dan lugar a diversos acuerdos comerciales que pueden influir en las prácticas periodísticas. Por tanto, la importancia del vínculo emocional del periodista deportivo con su profesión, que forma parte de su ideología profesional, se pone en práctica dentro de un contexto de una presión constante debido no solo a las demandas económicas sino también a las exigencias de generar grandes volúmenes de información, a menudo de forma inmediata y además para distintas plataformas. Paradójicamente, a pesar del reconocido impacto comercial del periodismo deportivo, los profesionales se enfrentan a otro elemento que añade complejidad a su trabajo: las malas condiciones laborales, especialmente en lo que respecta a los salarios. 
Los bajos ingresos son una característica promedio del periodismo en México. En un estudio del año 2008 realizado a nivel nacional se halló que el 70\% de los periodistas mexicanos recibían entre 0 y 10 mil pesos al mes [1]. De ellos, el 23\% solo percibía un máximo de 4 mil pesos mensuales (CIMAC, 2008: 21). En un informe posterior, correspondiente al tercer trimestre del año 2015, se encontró que el salario promedio de los periodistas en México era de 12,009 [2] pesos mensuales (Observatorio Laboral, 2015). Sin embargo, el salario no es lo único problemático en la situación laboral; un problema de base es la falta de reconocimiento a nivel legal, pues el periodismo en México ni siquiera es una actividad profesional reconocida en la Ley Federal del Trabajo (CIMAC, 2008: 28). Otros problemas como la falta de prestaciones laborales, horarios irregulares y una fuerte carga de trabajo que se ha acentuado con la tendencia de integración de redacciones en los medios de comunicación son algunos otros factores que pueden generar un ambiente de precariedad y con ello insatisfacción entre los periodistas. Este contexto laboral que rodea a los periodistas deportivos se relaciona de una manera compleja con su ideología profesional, la cual contempla una manera de adaptarse a estas condiciones de trabajo al apelar a los valores emocionales del periodista deportivo en relación a su actividad profesional, tal y como se ha identificado por otros investigadores en trabajos previos.

En un estudio sobre el estrés en periodistas deportivos llevado a cabo por Reinardy (2007), se encontró que éstos se sienten satisfechos con su trabajo a pesar de los conflictos que conlleva su profesión, siendo las tensiones entre la familia y el trabajo el asunto de mayor preocupación para ellos. La conclusión de Reinardy es que la tensión entre estos dos ámbitos puede generar estrés e insatisfacción laboral, sin embargo, los periodistas deportivos que formaron parte de la muestra de su estudio terminaron por priorizar sus deberes profesionales, ya que paradójicamente se sienten muy satisfechos con su trabajo, a pesar de las tensiones. Según sus hallazgos, los editores expresaron remordimiento por anteponer las exigencias del trabajo sobre las familiares, pero factores externos como cargas de trabajo, conflictos familiares y obstáculos organizacionales son equilibrados por un sentimiento interno de gozo, orgullo y éxito (Reinardy, 2007: 116). A diferencia de la investigación de Reinardy, en el presente estudio el conflicto principal de los periodistas deportivos es el de los salarios, aunque los periodistas tampoco desconocen los sacrificios familiares derivados de las obligaciones laborales.

Además de las complejas condiciones de trabajo que caracterizan al periodismo, los procesos de convergencia digital que comenzaron desde finales del Siglo XX, aún siguen ocasionando incertidumbre y cambios en la manera de pensar y ejercer la profesión. Diversos problemas e implicaciones de los distintos tipos de convergencia han sido tratados por los estudios sobre periodismo. Aunque en ocasiones puede creerse que la convergencia en el periodismo es sinónimo de integración de redacciones dentro de alguna organización periodística, en realidad abarca un espectro más amplio. Salaverría (2009) reconoce cuatro tipos de esta: convergencia tecnológica (enfocada en los dispositivos), convergencia empresarial (concentración), convergencia profesional (polivalencia) y convergencia de contenidos (multimedialidad). De estas, la convergencia profesional es de especial interés para el presente trabajo, ya que es un proceso que recae más en el periodista que el resto de las clases de convergencia (sin que sean ajenas al sujeto). Este tipo de convergencia se refiere a la polivalencia temática, funcional o 
mediática que ha caracterizado a los periodistas en años recientes, debido a que han adquirido la necesidad de trabajar temas más diversos, desempeñar más de una función y a menudo para varios tipos de medios o soportes. Esta polivalencia es un proceso que ha motivado diversas discusiones entre los periodistas, varias de las cuales son tratadas en las entrevistas que se realizaron para este trabajo.

\section{METODOLOGÍA}

La metodología del estudio se basó en un enfoque cualitativo para el cual se utilizó la técnica de entrevista semiestructurada como herramienta de investigación. Se eligió este camino para profundizar en los significados de los periodistas deportivos en relación a su ideología y conocer la manera en que asumen su profesión. Una de las ventajas de esta técnica es que permite entender el mundo desde la perspectiva del entrevistado y desmenuzar los significados de sus experiencias (Álvarez-Gayou, 2003: 109). A través de esta técnica, los sujetos de estudio fueron capaces de explicar detalladamente sus concepciones en torno a los valores profesionales de la cultura periodística en la que se desempeñan y en la cual se manifiestan valores con ciertas particularidades que, como hipótesis, podrían distinguirlos de otros colegas periodistas, como, por ejemplo, los periodistas políticos o generalistas. En las preguntas de investigación que se plantearon para guiar la investigación se aborda especialmente la función de los valores profesionales, los cuales legitiman legitimar las actividades de los periodistas y contribuyen en la construcción de una identidad profesional. Las preguntas de la investigación se plantearon de la siguiente manera:

P1: ¿Qué valores profesionales son los más relevantes para los periodistas deportivos de medios escritos de la Ciudad de México?

P2: ¿Cómo operan estos valores profesionales en un contexto influenciado por las presiones comerciales y condiciones de trabajo adversas?

P3: ¿Cómo perciben los periodistas deportivos su ideología profesional frente a un contexto de convergencia digital?

Las entrevistas se aplicaron cara a cara a 29 periodistas deportivos pertenecientes a trece distintos medios de comunicación ubicados en la Ciudad de México. Dichas entrevistas se concentraron en una muestra conformada por periodistas de medios impresos e Internet. Sin embargo, es sabido que las fronteras entre las distintas plataformas de los medios de comunicación son a menudo porosas, debido a los procesos de convergencia en distintos niveles. Esto quiere decir que es frecuente encontrar movilidad entre los periodistas deportivos, pues en ocasiones no solo trabajan para distintos soportes dentro de una misma organización sino también para distintos medios de comunicación.

Los 29 periodistas entrevistados pertenecen a distintos niveles jerárquicos, tanto ejecutivos como staffers según la clasificación de Breed (1955) con la finalidad de conocer diversas perspectivas sobre las rutinas de trabajo; es decir, la muestra incluye reporteros, editores, jefes de información y directores de medios deportivos. Los criterios de selección de los periodistas entrevistados respondieron a la búsqueda de una muestra diversa. La muestra de periodistas deportivos se fue construyendo a través del método conocido como "bola de 
nieve" (Hernández Sampieri et al, 2014: 388). Veintitrés de las entrevistas se llevaron a cabo en salas de redacción, cinco más en cafeterías cercanas a los lugares de trabajo de los periodistas y una en la casa de un reportero.

El método del estudio fue inductivo, esto quiere decir que las categorías emergieron de los testimonios recolectados durante el trabajo de campo (Sautu, 2007: 364) tal como ocurrió con la aparición del concepto de pasión y otros elementos de la cultura periodística que no provinieron de alguna hipótesis o categorías establecidas previamente. Antes de emprender el trabajo de campo se elaboraron algunas preguntas generales para todos los periodistas, aunque dada la flexibilidad de los estudios cualitativos se agregaron algunas otras cuestiones particulares. Las preguntas generales abordaron los siguientes temas: vocación del periodista, condiciones laborales, rutinas de trabajo, objetivos organizacionales y valores de la profesión. Una vez que se transcribió la información a de las 29 entrevistas se procedió a interpretar los datos ordenando las opiniones de los periodistas en distintas categorías analíticas, entre las cuales se encontraron: vínculo emocional con la profesión, valores normativos, estrategias de adaptación y problemas laborales.

En el siguiente apartado se describirán los hallazgos construidos a partir del análisis de los datos cualitativos que surgieron a de las entrevistas y charlas informales off the record con periodistas durante las visitas a las salas de redacción de los medios mencionados anteriormente. Debido a la complejidad del periodismo deportivo y a la riqueza de los métodos cualitativos se encontraron más hallazgos de los mencionados en las categorías y subcategorías, pero para efectos del presente estudio solo me enfocaré en lo que respecta a las preguntas de investigación. Finalmente, para cumplir con un acuerdo de confidencialidad con los participantes no se mencionan los nombres de los 29 periodistas que aceptaron colaborar en el estudio, aunque a continuación se muestra la relación de periodistas entrevistados por medio. 
http://dx.doi.org/10.12795/Ambitos.2018.i40.13

\begin{tabular}{|c|c|c|}
\hline Identificador & Puesto & Medio y soporte \\
\hline Reportero 1 & Reportero & Expansión (impreso y web) \\
\hline Editor 1 & Editor & Mediotiempo (web) \\
\hline Jefe 1 & Jefe de información & Mediotiempo (web) \\
\hline Reportera 1 & Reportera & Proceso (impreso y web) \\
\hline Reportero 2 & Reportero y columnista & Yahoo (web) \\
\hline Editor 2 & Editor & Milenio/La Afición (impreso, TV y web) \\
\hline Reportera 2 & Reportera & Milenio/La Afición (impreso, TV y web) \\
\hline Director 1 & Director & Milenio/La Afición (impreso, TV y web) \\
\hline Reportero 3 & Reportero & Agencia Notimex (web) \\
\hline Editor 3 & Editor & El Economista (impreso y web) \\
\hline Reportero 4 & Reportero & El Economista (impreso y web) \\
\hline Reportero 5 & Reportero & Grupo Imagen (impreso, TV y web) \\
\hline Editor 4 & Editor & Grupo Imagen (impreso, TV y web) \\
\hline Reportero 6 & Reportero & Grupo Imagen (impreso, TVy web) \\
\hline Editor 5 & Editor & Grupo Imagen (impreso, TV y web) \\
\hline Reportero 7 & Reportero & Cancha (impreso y web) \\
\hline Reportero 8 & Reportero & Cancha (impreso y web) \\
\hline Reportero 9 & Reportero & Tiempo Real (web) \\
\hline Reportero 10 & Reportero & Récord (impreso y web) \\
\hline Editor 6 & Editor & Récord (impreso y web) \\
\hline Reportero 11 & Reportero & Récord (impreso y web) \\
\hline Reportero 12 & Reportero & Récord (impreso y web) \\
\hline Reportero 13 & Reportero & Récord (impreso y web) \\
\hline Subdirector 1 & Subdirector & Récord (impreso y web) \\
\hline Editor 7 & Editor & Esto (impreso y web) \\
\hline Director 2 & Director & Esto (impreso y web) \\
\hline Reportera 2 & Reportera & Terra (web) \\
\hline Editor 7 & Editor & El Universal (impreso y web) \\
\hline Reportero 14 & Reportero & El Universal (impreso y web) \\
\hline
\end{tabular}

\section{RESULTADOS}

\subsection{La pasión como valor profesional dominante}

En su acepción aristotélica, el pathos se refiere a los sentimientos que pueden ser utilizados como parte de una argumentación (Rodríguez, 2005). En este sentido, puede decirse que los periodistas deportivos apelan al pathos no solo para mover las pasiones de su auditorio (Arroyas \& Berná, 2015: 151) sino que también utilizan las emociones para legitimar su discurso profesional y justificar sus decisiones. Se propone que esta noción es un valor profesional dominante porque emergió de las entrevistas especialmente cuando se les preguntó a los periodistas “¿Qué características debería tener idealmente un periodista deportivo?". Al responder este cuestionamiento apareció en varias conversaciones la idea de que el periodista deportivo debe tener pasión por su profesión; aunque esta categoría también se mencionó a partir de otras preguntas en varias 
entrevistas. Al tratarse de un valor altamente subjetivo, la objetividad en el periodismo deportivo parece ser una condición distante en la práctica, la cual no aparece como un valor relevante en las entrevistas realizadas. Como ha ocurrido en estudios similares sobre periodismo deportivo, este distanciamiento con la objetividad "contrasta con las normas tradicionales de objetividad que son esenciales en otras áreas de las redacciones" (English, 2016: 1). Aunque frecuentemente aparece en slogans de medios deportivos, la pasión por el deporte es más que un elemento publicitario porque cumple funciones importantes en la cultura del periodismo deportivo. Esta función tiene que ver con la noción de cemento cultural porque se trata de un valor que da sentido y une a los periodistas deportivos como grupo. Como señaló uno de los editores entrevistados:

Para mí el deporte es pasión. Tendría que hablar con un periodista de política, seguramente tiene una pasión por lo que hace, pero aquí es una pasión por el deporte. Una pasión 24 horas. En serio, no te miento, los que nos dedicamos a esto, sabemos que todo el día hay deporte, yo amanezco viendo deporte y duermo viendo deporte. (Editor 1).

Por otra parte, este valor profesional no solo une a los periodistas ya activos, sino que puede ser visto también como un requisito de entrada a este ámbito laboral para los jóvenes que buscan dedicarse a la actividad. Una de las principales aptitudes que buscan los jefes o quienes participan en la contratación de nuevos periodistas en medios deportivos es que los aspirantes tengan una relación afectiva con los deportes. Después de esto, aparecen otras condiciones deseables entre sus criterios de contratación, pero la emotividad por el deporte (más que por el periodismo) es una de las principales características ideológicas en esta cultura. Siguiendo a Deuze (2005) este valor profesional puede ser concebido como un 'cemento cultural' que mantiene unidos a los periodistas, a pesar del estrés y de los sacrificios familiares que caracterizan a esta actividad (Reinardy, 2007).

Otros valores profesionales normativos entre los periodistas deportivos entrevistados tienen que ver con el conocimiento acerca del deporte. En concreto, de acuerdo con algunos editores y reporteros entrevistados, el periodista deportivo está obligado a conocer más de deporte que el público. Esto implica que el periodista deportivo debe poseer un acervo de datos superior al de sus lectores, aunque también es importante que esté familiarizado con todos los deportes y no únicamente con futbol, esto para cumplir las exigencias de los lectores de algún nicho como los aficionados al futbol americano, basquetbol, béisbol u otras disciplinas apartadas de la gran cobertura que recibe el futbol. Así lo verbalizó uno de los editores generales entrevistados al preguntarle sobre el perfil de jóvenes que pretende tener en su redacción:

Lo primero que vemos es que estén interesados en deportes, no sólo en futbol. Pasa mucho y más ahora que todos están enterados de futbol internacional, todos saben de Barcelona y Real Madrid, pero poca gente tiene conocimiento de tenis, futbol americano, etc. Entonces si no están muy clavados por lo menos que estén abiertos. (Editor 2).

Algunos otros periodistas agregaron que idealmente quienes se dedican a esta profesión no únicamente deben ser expertos conocedores en deporte, sino que también deben contar con lo que denominan "cultura general". Es decir, tener noción de las noticias que acontecen en los ámbitos político, económico o social. Lexias como "saber a cuánto está el 
dólar", o "saber qué está pasando en el mundo" fueron utilizadas para ilustrar esta idea de que el periodista deportivo no debería concentrarse únicamente en el deporte, sino que también debe estar al tanto de otros temas informativos.

Se identificó que los valores de la pasión y el conocimiento (en ese orden) son los dos aspectos más relevantes para los periodistas deportivos entrevistados. Otros valores normativos que también fueron mencionados en diversas entrevistas tienen que ver con habilidades prácticas como la buena redacción y ortografía, así como contar con destrezas multitask o multimedia, con la idea de adaptarse a las necesidades de la convergencia profesional. En cambio, aspectos poco mencionados entre los valores deseables por parte de los sujetos de estudio fueron la ética y la capacidad para investigar. En este sentido, una de las reporteras entrevistadas ligó estos dos elementos debido a que mantener un distanciamiento con las fuentes permite ejercer la crítica y el periodismo de investigación:

Ser buen periodista deportivo tiene varias aristas. Una, tiene que ver con cómo eres tu como persona: tu ética, tu integridad, que yo creo que es muy difícil, pero tienes que ser una persona fiel a tus principios en el sentido de que no aceptes regalos, dinero. Que nunca escribas mal de alguien por venganza, o que hables bien de alguien porque es tu amigo. Tienes que tener claro que no puedes ser amigo de los deportistas, entrenadores, directivos, ni de nadie. Sí te puedes llevar bien con ellos, tienen que ser tu fuente, pero el día que les encuentres algo lo tienes que publicar, no te lo puedes guardar. (Reportera 1).

En este apartado puede concluirse que el valor normativo principal dentro de la cultura de los periodistas deportivos es el que los sujetos verbalizaron como pasión, un concepto que tiene que ver con aspectos emocionales que ligan a los periodistas deportivos con su profesión. Otro valor relevante pero secundario para ejercer el periodismo deportivo es el conocimiento, y finalmente, de importancia relativa, valores relacionados con habilidades pragmáticas, así como la ética y la investigación (lo cual conlleva el ejercicio de un periodismo critico) forman parte del perfil ideal por parte de los periodistas entrevistados.

\subsection{Operacionalización de los valores profesionales en el periodismo deportivo}

Además de tener una función cohesiva entre los periodistas, los valores profesionales pueden materializarse con distintos fines en las rutinas de trabajo. Por la manera en que los sujetos de estudio explicaron su percepción del periodismo deportivo, puede decirse que otras funciones relevantes del valor normativo llamado pasión son las de compensar las carencias laborales y adaptarse a las exigencias de la profesión. Los periodistas deportivos entrevistados expresaron que la afición o gusto por el deporte que suelen tener justifica a menudo las razones por las que se dedican a esta profesión y a su vez el por qué se mantienen ejerciendo esta actividad a pesar de que no gozan de condiciones favorables en cuanto a salarios o tiempos libres. Si bien las condiciones de trabajo adversas no son exclusivas de las redacciones deportivas, lo distintivo en este ámbito es que el vínculo entre periodista y profesión da lugar a un mecanismo autodisciplinario en el cual el periodista desafía las adversidades laborales a partir de su lazo emocional, lo que le permite desempeñarse como un buen periodista deportivo en términos de profesionalismo de acuerdo con los valores normativos dominantes en su actividad. Lo anterior puede apreciarse en las siguientes opiniones recopiladas en las entrevistas: 
Dentro del periodismo uno aprende a lidiar con la frustración. Si estás en esto es porque tienes capacidad para lidiar con este tipo de cosas. Te vas adaptando a lo que te van presentando, y tienes la capacidad de rearmarte y seguir adelante. Eso es en todo el periodismo no sólo el deportivo. (Reportera 2).

Te digo algo que no me pesa. Disfrutas el periodismo deportivo porque el día que me tocó ir a la Arena Ciudad de México a cubrir una pelea, cuando llegas a la Arena dices... ¿Cómo paga uno por estar en el lugar en el que siempre quiso estar? ¿Cómo pago el estar en la función de Márquez, en una convención, en todo lo que he podido disfrutar como periodista? Eso nadie me lo quita. El periodista deportivo vive de los grandes eventos. (Reportero 2).

Testimonios como el del Reportero 2 muestran que, si bien el periodismo deportivo implica adversidades, también ofrece una compensación a éstas a través de aspectos de la profesión que son bien valorados entre los periodistas, como lo son los viajes o la cobertura de grandes eventos deportivos. Desde una visión crítica, estaríamos ante un mecanismo de control que opera en dos sentidos. Como argumenta Soloski (1989), existen dos maneras en las que las organizaciones periodísticas establecen una estrategia de control por medio del profesionalismo. La primera, al establecer estándares y normas de comportamiento; y la segunda, mediante un sistema de recompensas. Bajo esta postura crítica, los valores profesionales normativos formarían parte de las pautas de comportamiento, entre las que destaca una relación pasional con el deporte. Por otro lado, considerando que el periodismo no goza de un estatus alto en términos económicos para quienes lo ejercen, la cobertura de ciertos eventos puede ser vista como una clase de recompensa para ellos y al mismo tiempo señal de crecimiento en cuanto a la jerarquía profesional.

Las siguientes opiniones muestran cómo los valores profesionales van ligados a las pautas de comportamiento deseables dentro del periodismo deportivo. Uno de los reporteros de un diario especializado expresó que la pasión es un valor importante no solo en el periodismo sino en el deporte-espectáculo en general:

"El periodismo deportivo necesita una gran dosis de pasión como el mismo deporte exige. El deporte tiene tres condicionantes: el deporte es espectáculo, es agradable. Es energético; provoca que tengamos energía. Cada vez que hay una competición de carácter internacional como Mundiales, los chicos en las calles salen y se sienten cracks del universo. Y también por supuesto el deporte es formativo y aspiracional” (Reportero 13).

Siguiendo, uno de los editores manifestó de forma autocrítica que la ausencia de este valor puede ocasionar resultados negativos en el desempeño de los periodistas, ya sean reporteros o editores. Además, explicó desde su punto de vista la relación que existe entre lo emocional con el trabajo de los periodistas deportivos:

"Creo que los editores carecemos de imaginación y los reporteros de propuestas. Habría que cuestionarnos si hay pasión por lo que hacemos, entendiendo el concepto como una motivación para ser mejor y más profesional. Creo que deberíamos de enfocar las 
motivaciones a no perder la pasión. Eso genera que estés metido, que tengas curiosidad para buscar datos, historias, enfoques. Te despierta la mente y te hace decir, a ver de qué podemos hablar que no se haya hablado. ¿Cómo podemos investigar más?”. (Editor 3).

Como mencionan los entrevistados, el gusto y la pasión por el deporte no solo funcionan a nivel simbólico dentro de la ideología profesional, sino que en términos pragmáticos también encuentran una materialización en las rutinas de trabajo al ser un punto de partida para ejercer el periodismo de forma adecuada según su perspectiva. Bajo este supuesto de los sujetos de estudio, el gusto por el deporte permite que el periodista deportivo se involucre intensamente con su profesión. Las opiniones referidas de los entrevistados muestran que no solo se busca que los periodistas estén al tanto de la información de actualidad, sino que tengan un lazo emocional con el deporte y en consecuencia con el periodismo deportivo.

Por otra parte, en lo que se refiere al sistema de recompensas dentro del periodismo deportivo, los viajes y la cobertura de ciertos eventos tienen un significado especial. Esta clase de coberturas suelen ser vistas como una muestra de crecimiento profesional por parte de los periodistas y en un par de casos, los periodistas entrevistados mencionaron que viajar es lo que más les gusta de su actividad. Las únicas dos excepciones fueron por parte de dos periodistas veteranos, quienes manifestaron sentirse cansados a su edad para someterse al esfuerzo que implica viajar para realizar alguna cobertura. En cambio, así se expresaron dos periodistas que reconocen la relevancia de los viajes de trabajo en este ámbito profesional. La primera opinión corresponde a un periodista joven, de 25 años, y la segunda a un editor de 35 años de edad:

Lo que me llena de mi profesión es encontrar historias, ir a cubrir eventos. Me emociona mucho cuando me mandan a Las Vegas, a unos Juegos Olímpicos [...] Conocer a los protagonistas y contar historias me emociona mucho [...] Hay que saber hasta dónde puedes ceder. Soy soltero, tengo una buena edad, todavía puedo darme ciertas libertades de no tener una entrada económica tan alta. (Reportero 14).

Por méritos se va viendo quiénes viajan y quiénes no. Cuando se autoriza el viaje, se da como un premio a una cobertura anterior, para tener una motivación. Esto nos gusta porque puedes estar presente en el lugar de los hechos. Hay gente que al igual que un jugador va cumpliendo un proceso. Para nosotros los procesos se cumplen por coberturas, el que cubrió un Mundial de futbol cumple un ciclo. Hemos tenido ese proceso de que un reportero que fue y cubrió el Mundial ahora trabaja como coeditor o editor. (Editor 7).

En síntesis, el valor profesional dominante dentro del periodismo deportivo cumple un rol que permite a los periodistas compensar sus carencias y al mismo tiempo sentirse recompensados. Incluso, el solo hecho de ser periodista deportivo ya es visto como una recompensa para algunos, debido a la gran demanda de aspirantes. Por otro lado, los viajes de cobertura son otra recompensa que fortalece el lazo entre periodista y profesión, especialmente si se trata de una persona joven, pero que además funge como un indicador de estatus al interior o al exterior de una organización periodística.

\subsection{El sólido "cemento cultural” ante la convergencia digital}

A pesar de la incertidumbre que se ha generado -entre otros factores- a raíz de los 
procesos de convergencia digital en las culturas periodísticas, existen continuidades en las formas de pensar y de ejercer el periodismo. Es decir, no todo ha cambiado, o al menos, no por completo. Si bien la identidad profesional, al igual que todos los factores que forman parte de una cultura, tiene un carácter dinámico, esto no quiere decir que sus cambios ocurran demasiado rápido o que partan de una base ahistórica. Como advierte Tumber (2014:74), "es incorrecto pensar que las tecnologías digitales han erradicado todas las relaciones previamente existentes detrás de las estructuras de los medios". En el caso del presente estudio, se pudo observar que los periodistas deportivos acarrean prácticas y valores de amplia tradición construidos a través del tiempo que siguen vigentes en la denominada era digital. Uno de esos valores que justamente se mantiene como un referente en este contexto es la manera emocional a partir de la cual comprenden su profesión.

Las entrevistas realizadas arrojaron que las principales modificaciones en el ejercicio periodístico que perciben los periodistas deportivos tienen que ver con aspectos prácticos de su trabajo como la manera de obtener y difundir información. Algo que les genera incertidumbre es el hecho de que a causa del desarrollo de las redes sociales se ha puesto en debate lo que significa ser un periodista profesional, lo que puede afectar su estatus. Esto ha motivado reflexiones sobre lo que implica el tener que convivir con blogueros, youtubers o aficionados que difunden información deportiva con un alcance significativo dentro del mismo campo periodístico. La siguiente opinión fue expresada por uno de los reporteros de un diario de referencia con respecto a las redes sociales y la credibilidad en el ejercicio periodístico:

A mí esto del Internet y redes sociales se me hace una gran ayuda, pero no tienes que depender de eso ni darle demasiada credibilidad al Twitter porque ya cualquiera opina. Yo como reportero escribo algo ahí y a lo mejor alguna gente me conoce y es creíble, pero si no fuera reportero, si fuera una persona equis también lo puedo hacer y es creíble para mucha gente. Hay que tomar con pinzas esto de las redes sociales, es un boom que a veces se sale de control. Debemos darle su dimensión justa y no creerle siempre, porque luego resulta que tienes que desmentir, decir que no, y a uno lo vuelve loco. Debe servir como apoyo, pero no una vía para hacer tu trabajo. (Reportero 7).

Finalmente, la inmediatez es otra de las grandes preocupaciones de los periodistas en el contexto digital debido a dos problemas principalmente: el primero tiene que ver con la difusión de información imprecisa derivada de la velocidad con la que se produce y propaga, y el segundo problema, es que por estos mismos ritmos de trabajo acelerados se ha vuelto más difícil investigar. Sin embargo, esto no solo puede afectar la producción de la noticia sino también su estatus como periodistas, ya que pueden llegar a sentirse meros agregadores de información más que reporteros, como explica uno de ellos:

No se investiga porque los medios son ya muy rápidos, sobre todo Internet porque subes una noticia y se triplica. Por ejemplo, lo del maratón de Boston; te enteras de que hubo bombas, y a los cinco minutos te bombardean de información. Y tú obviamente no estás en Boston, estás en México y lo que haces es alimentar tus páginas de lo que dicen los cables. A lo mejor uno o dos medios que estuvieron en el maratón son los que investigaron y todos los demás empiezan a repetir de lo que jalaron de los principales y tú en lugar de ser periodista te conviertes en receptor nada más. 
Independientemente de los problemas relacionados con la presión por obtener clics, la inmediatez o la escasa investigación en medio de procesos convergentes, el "cemento cultural" que une a los periodistas deportivos se mantiene sólido. Más bien, los problemas actuales relativos a la forma en la que los entrevistados perciben la profesión tienen que ver con la acumulación de trabajo, las nuevas competencias necesarias, la responsabilidad y el rigor al difundir la información noticiosa. Un joven reportero de un portal de Internet opinó lo siguiente cuando se le preguntó por las características que debe tener un periodista deportivo en un contexto digital:

Creo que estar comprometido es fundamental. Si no te gusta o te da flojera, yo diría que mejor te busques otra cosa porque vas a sacrificar fines de semana, Navidad. Por ejemplo, en año nuevo me tocó trabajar y ni modo. Me tocó la guardia, salí antes, pero al otro día, el 1 de enero a las 10:00 ya tenía que estar trabajando. En mi caso, los deportes siempre me gustaron, me enamoro muy fácil de cualquier deporte, así sea nuevo. Conocí el futbol, dije "esto es increíble".; conocí el rugby y también le intentaba. Nunca fui de los que desde chicos dijeron "yo quiero ser periodismo deportivo", pero de repente vi que esa parte me gustaba mucho y lo descubrí ya en la preparatoria.

\section{CONCLUSIONES}

Según las percepciones de los periodistas deportivos entrevistados, el valor dominante de su actividad es verbalizado como pasión hacia el deporte y hacia su profesión. Este concepto se materializa en aspectos como la perseverancia, el gusto por el deporte, la capacidad de aprender y la adaptación a condiciones adversas. A través de este valor, los periodistas deportivos adquieren autodisciplina y al mismo tiempo compensan las deficientes condiciones de trabajo y los sacrificios personales que implica su trabajo, haciendo que se sientan orgullosos de ser periodistas deportivos. Pese a estos desafíos, los periodistas se mantienen ejerciendo la profesión con la ayuda de recompensas distintivas de su entorno: la primera es el hecho mismo de ser periodista deportivo y la segunda, los viajes para la cobertura de grandes eventos. La identidad del periodista deportivo se sostiene en un fuerte vínculo del periodista con su profesión, el cual se expresa a través de la ideología profesional. De manera secundaria, aparecieron en las entrevistas valores como la ética, la responsabilidad, la honestidad, y otras habilidades como la buena redacción y la capacidad de investigar.

Frente a los desafíos de la convergencia digital, el valor dominante de los periodistas deportivos se mantiene como "cemento cultural" al ser mencionado como una pauta de comportamiento aún bajo el fenómeno de la digitalización. Más bien, la incertidumbre de los periodistas deportivos no radica en los valores normativos tradicionales sino en aspectos que quizás en un contexto análogo (no digital) no tendrían tanta relevancia, como la ética, el rigor, la responsabilidad o la verificación, todo esto ante la facilidad con la que se puede propagar información falsa a raíz de la inmediatez de los medios digitales. Siguiendo, el ritmo de la inmediatez no solo facilita la generación de información imprecisa, sino que también ha afectado la posibilidad de verificar e investigar.

El presente trabajo buscó comprender algunos aspectos relevantes de la ideología profesional, aunque solo se concentró una pequeña muestra de la cultura del periodismo deportivo. Al tratarse de un periodismo temático relacionado con fuertes influencias como 
son la comercialización y la comercialización, es pertinente indagar sobre aspectos ideológicos de quienes lo ejercen, aunque también es necesario ampliar esta clase de estudios no solo en términos geográficos sino con la intención de vincular estos hallazgos con los de otros periodismos temáticos. Por otra parte, también hay que considerar el dinamismo de los aspectos culturales de la profesión periodística, advirtiendo que el análisis de temas relacionados con la ideología o la identidad depende de un contexto específico, sin obviar los cambios y continuidades del ejercicio del periodismo.

\section{REFERENCIAS BIBLIOGRÁFICAS}

ÁLVAREZ-GAYOU, J. (2003): ¿Cómo hacer investigación cualitativa? Fundamentos y metodología. México: Paidós Educador.

ANDREWS, P. (2006): Sports journalism: a practical guide. Londres: Thousand Oaks.

BENSON, R. \& NEVEU, E. (eds.) (2005): Bourdieu and the journalistic field. Cambridge: Polity Press.

BOYLE, R. (2006): Sports Journalism: Context and Issues. Londres: Sage.

BREED, W. (1955): Social Control in the Newsroom. En BERKOWITZ, D. (ed.) (2011): Cultural meanings of news. Londres: Sage, (pp. 326-355)

CIMAC (2008): Condiciones laborales de los y las periodistas en México, un acercamiento. México: CIMAC.

DEUZE, M.: "What is journalism? Professional identity and ideology of journalists reconsidered”. Journalism, vol. 6 (Noviembre 2005), n4, pp. 442-464.

ENGLISH, P.: "Cheerleaders or critics? Australian and indian sports journalists in the contemporary age”. Digital Journalism, vol. 5 (Agosto 2016), n5, pp. 532-548.

ENGLISH, P.: "Mapping the sports journalism field: Bourdieu and the broadsheet newsrooms”. Journalism, vol. 17 (Abril 2015), n8, pp. 1001-1017.

GARRISSON, B. \& SALWEN, M.: "Professional orientations of Sports journalists". Newspaper Research Journal, vol. 10 (Verano/Otoño 1989), n4, pp. 77-84.

GARRISSON, B. \& SALWEN, M.: "Finding their place in journalism: newspaper sports journalists and their professional problems". Journal of Sport and Social Issues, vol. 22 (Febrero 1998) n¹, pp. 88-102.

GRUBENMANN, S. \& MECKEL, M.: "Journalist's professional identity. A resource to cope witch change in the industry? Journalism Studies, vol. 18 (2015), nº 6, pp. 732-748.

HANITZSCH, T. "Deconstructing Journalism Culture: Toward a Universal Theory”. Communication Theory, vol. 17 (Octubre 2007), n4, pp. 367-385.

HARDIN, M., ZHONG, B. \& WHITESIDE, E. (2009): “Sports coverage: 'Toy department' or public-service journalism? The relationship between reporter's ethics and attitudes toward the profession". International Journal of Sport Communication, vol. 2 (Septiembre 2009), n³, pp. 319-339. 
HENNINGHAM, J.: "A profile of Australian sports journalists". ACHPER Healthy Lifestyles Journal, vol. 42 (1995) n³, pp. 13-17.

HERNÁNDEZ-SAMPIERI, R., FERNÁNDEZ-COLLADO, C. \& BAPTISTA, P. (2014): Metodología de la investigación. México: McGraw Hill.

KNOTT, D., CARROLL, V. \& MEYER, P.: "Social responsibility wins when CEO has been editor”. Newspaper Research Journal, vol. 23 (Enero 2002), n¹, pp. 25-37.

LANGE, K. (2002): Sport and new media: A profile of internet sport journalists in Australia. Tesis doctoral inédita, Victoria University, Australia.

OLLER, M. \& MEIER, K. (2012): La cultura periodística de España y Suiza. Madrid: Editorial Fragua.

NICHOLSON, M., ZION, L. \& LOWDEN, D.: "A Profile of Australian Sports Journalists (revisited)”. Media International Australia, vol. 140 (Agosto 2011), n¹, pp. 84-95.

REESE, S. (2001): Understanding the global journalist: a hierarchy-of-infuences approach. En BERKOWITZ, D. (ed.) (2011): Cultural meanings of news. Londres: Sage, (pp. 173187).

REINARDY, S.: "Satisfaction vs Sacrifice: Sports Editors asses the influences of life issues on job satisfaction”. J\&MC, vol. 84 (Primavera 2007), n¹, pp. 105-121.

RODICA, M.: "The influence of new technologies on journalism practices". Jurnalism si comunicare, vol. 48 (2015) n², pp. 25-31.

RODRÍGUEZ, L.: “Ética argumentativa en Aristóteles”. Revista Digital Universitaria, vol. 6 (Marzo 2005), n³, pp. 1-40.

ROWE, D.: "Sports journalism: Still the 'toy department' of the news media?" Journalism, vol. 8 (Agosto 2007), n4, pp. 385-404.

SALAVERRÍA, R.: Actas del I Congreso Internacional de Ciberperiodismo y Web 2.0. celebrado en Bilbao del 11 al 13 de noviembre de 2009.

SANTIAGO, A. (2001): El nacimiento del periodismo deportivo en México. Tesis inédita de licenciatura, UNAM, Ciudad de México.

SAUTU, R. et al. (2006): Manual de metodología. Buenos Aires: CLACSO.

SCHUDSON, M. (2003): The sociology of news. Nueva York: W.W. Norton.

SOLOSKI, J.: "News reporting and professionalism: Some constraints on the reporting of the news”. Media, Culture \& Society, vol. 11 (Abril 1989), n², pp. 207-228.

VAN ZOONEN, L.: "A professional, unreliable, heroic marionette. Structure, agency and subjectivity in contemporary journalisms". European Journal of Cultural Studies, vol. 1 (Enero 1998), n¹, pp. 123-143. 
[1] El dólar estadounidense inició el 2008 con un valor de 10.91 pesos mexicanos y cerró el año costando 13.77 pesos.

[2] En diciembre de 2015, el equivalente de 12,009 pesos mexicanos era de 706 dólares estadounidenses.

\section{BREVE SEMBLANZA DEL AUTOR:}

El Mtro. Juan Carlos Colin Vaughan (Ciudad de México, 1983) es egresado de la Licenciatura en Comunicación de la Universidad Iberoamericana Ciudad de México y Maestro en Periodismo por la Universidad de Buenos Aires. Actualmente es estudiante del Doctorado en Comunicación de la Universidad Iberoamericana. Se especializa en temas referentes al Periodismo y el Deporte.

- Recibido: 24/09/2017

- Aceptado: 11/03/2018

Ámbitos. Revista Internacional de Comunicación, n.40, edición de primavera, 2018. 